http://jmscr.igmpublication.org/home/

ISSN (e)-2347-176x ISSN (p) 2455-0450

crossref DOI: https://dx.doi.org/10.18535/jmscr/v8i7.97

Journal Of Medical Science And Clinical Research

IGM Publication

An Official Publication of IGM Publication

\title{
Small Intestine Perforation as Initial Presentation of Granulomatosis with Polyangiitis
}

\author{
Authors \\ Firdevs ULUTAŞ ${ }^{1 *}$, Tuğba AYTEKİN ${ }^{2}$, Özgür ERAY ${ }^{2}$, Veli ÇOBANKARA ${ }^{1}$, \\ Mevlüt ÇERI' ${ }^{3}$, Ŭgur KARASU ${ }^{1}$
}

${ }^{1}$ Department of Rheumatology, Pamukkale University Faculty of Medicine, Denizli, TURKEY

${ }^{2}$ Department of Internal Medicine, Pamukkale University Faculty of Medicine, Denizli, TURKEY

${ }^{3}$ Department of Nephrology, Pamukkale University Faculty of Medicine, Denizli, TURKEY

*Corresponding Author

Firdevs ULUTAŞ

Department of Rheumatology, Pamukkale University Faculty of Medicine, Denizli, TURKEY

\begin{abstract}
Granulomatosis with polyangiitis (GPA) is necrotising vasculitis of small and medium sized blood vessels. GPA typically affects the upper/lower respiratory system and kidneys. Gastrointestinal system involvement is rarely seen. In the present paper, we report a case of small intestinal perforation as a presenting clinical manifestation in a patient with systemic GPA. The patient initially underwent surgical repair, and than achieved remission with methylprednisolone and cyclophosphamide.
\end{abstract}

\section{Introduction}

Granulomatosis with polyangiitis (previously defined as Wegener's granulomatosis) (GPA) is a small vessel vasculitis characterised by granulomatous necrotising inflammation ${ }^{(1)}$. Gastrointestinal system (GIS) involvement is seen in $10 \%$ to $24 \%$ of patients with GPA and is more frequently revealed in the first 1 or 2 years after the diagnosis of $\mathrm{GPA}^{(2)}$. In the analysed group of 34 GPA patients, 9 of them $(26 \%)$ had gastrointestinal manifestations and 5 of them had GIS symptoms as a initial presentation ${ }^{(3)}$. Here, we report a case of small intestinal perforation as a presenting clinical manifestation in a patient with systemic GPA.

\section{Case Presentation}

A 39-year-old man was diagnosed with progressive diffuse multisystemic GPA, affecting lungs, upper respiratory system, joints and kidneys in our tertiary health center. In the recent two months, he initially presented with gastrointestinal symptoms including vomiting and acute abdomen. It was detected that contrastenhanced computerized tomography scanning of the abdomen revealed thickening of the small intestine wall, air-fluid levels and generalized peritonitis due to ileal perforation. Promptly laparoscopic small intestine resection has been done. The pathological specimens revealed nonspecific diffuse inflammatory changes and neutrophilic microabscesses in the small intestine wall. 


\section{JMSCR Vol||08||Issue||07||Page 591-594||July}

Currently he presented with a constitutional symptoms likewise fatigue, fever and epistaxis. On physical examination, a saddle nose deformity and mild pretibial oedema were observed. Laboratory findings showed: white blood cell counts:15.750 $\mathrm{K} / \mu \mathrm{L}$ (neutrophils: $86.0 \%$ ), hemoglobin:8.3 g/dL, platelet count: 651.000 $\mathrm{K} / \mu \mathrm{L}$, C-reactive protein: $204 \mathrm{mg} / \mathrm{L}$, erythrocyte sedimentation rate: $91 \mathrm{~mm} /$ hour, creatinine: 2.4 $\mathrm{mg} / \mathrm{dL}$, albumin: $29 \mathrm{~g} / \mathrm{L}$ and 24-hour urine protein excretion: $2200 \mathrm{mg} /$ day. Serological tests showed a positive result for an antineutrophil cytoplasmic antibody (ANCA) specific for proteinase-3 (PR3).

X-ray and computed tomography (CT) of chest revealed a cavitary nodul in right lung field. Paranasal sinus CT showed septum perforation, soft mass lesions in the left maxillary sinus and also thickening lateral wall of left maxillary sinus. The kidney biopsy revealed a crescentic glomerulonephritis. Remission was achieved with induction therapy including steroid pulse and intravenous cyclophosphamide. The patient's gastrointestinal and upper respiratory system symptoms disappeared. Renal function tests and acute phase reactants improved at the follow-up. He did not require dialysis, plasma exchange or another surgical intervention.

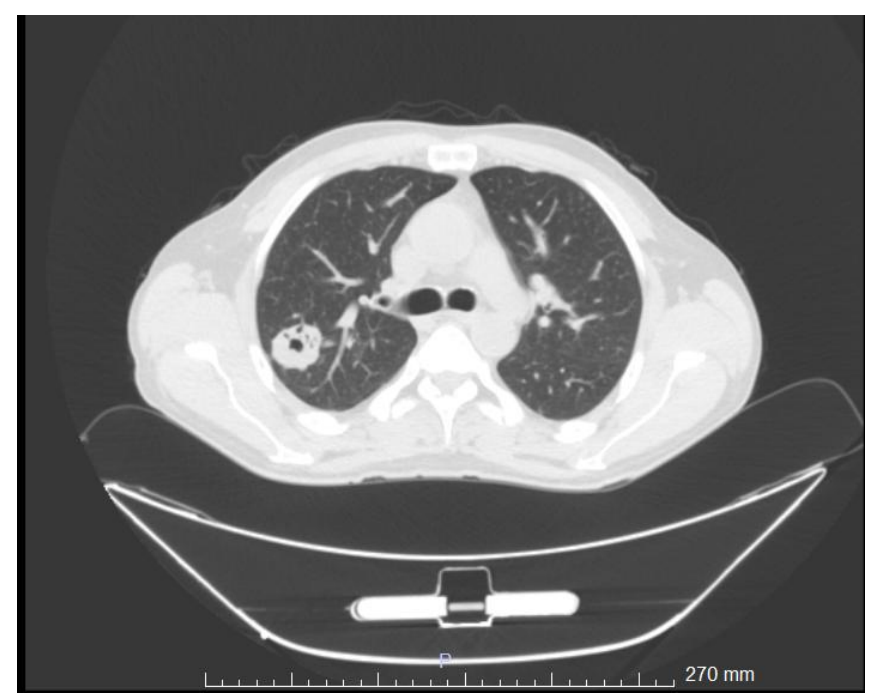

Computed tomography of lungs: a cavitary nodul in right lung

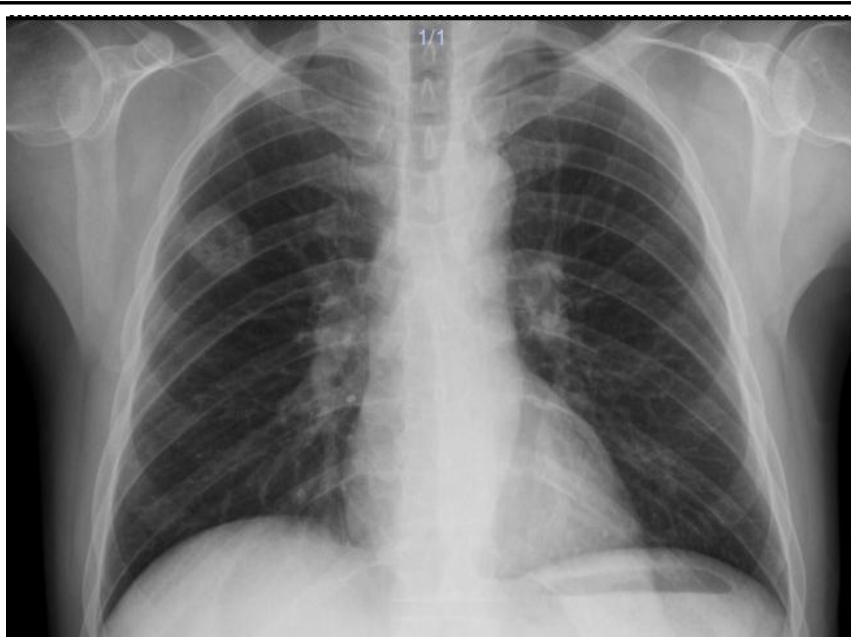

Postero-anterior chest X-ray: a cavitary nodul in right lung field

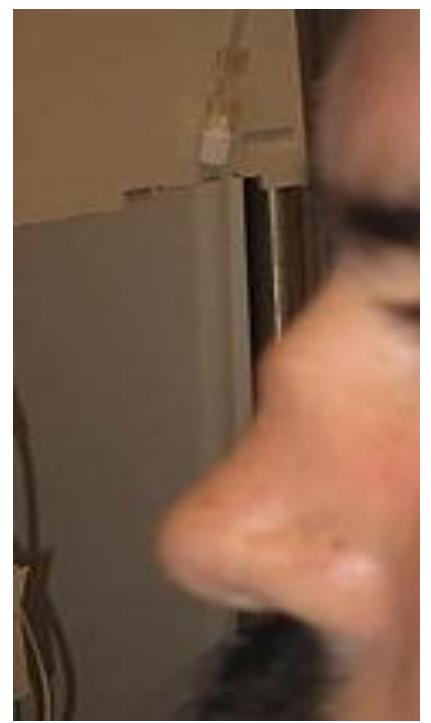

A saddle nose deformity on physical examination

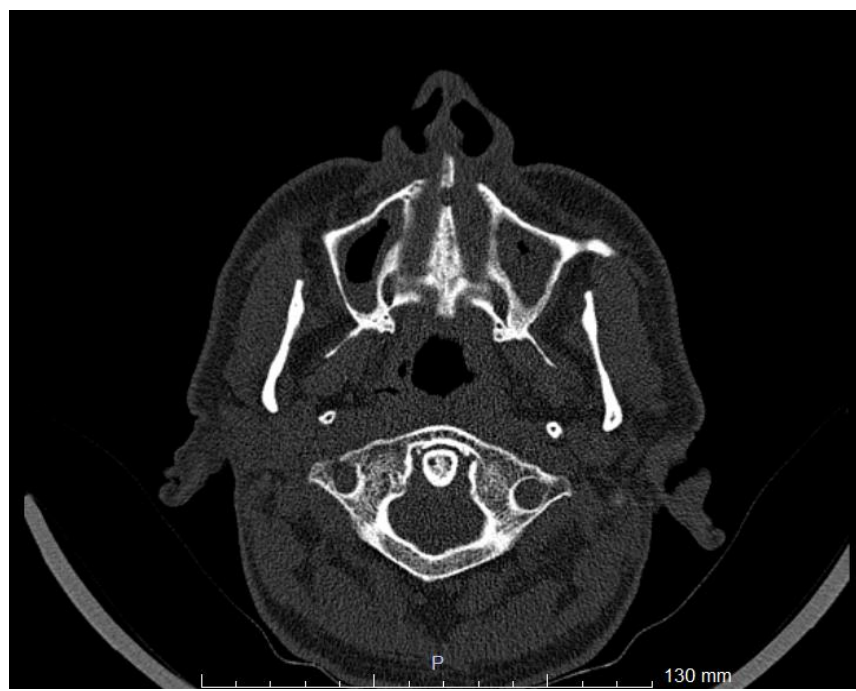

Computed tomography of paranasal sinuses: septum perforation, soft mass lesions in the left maxillary sinus and thickening lateral wall of left maxillary sinus 


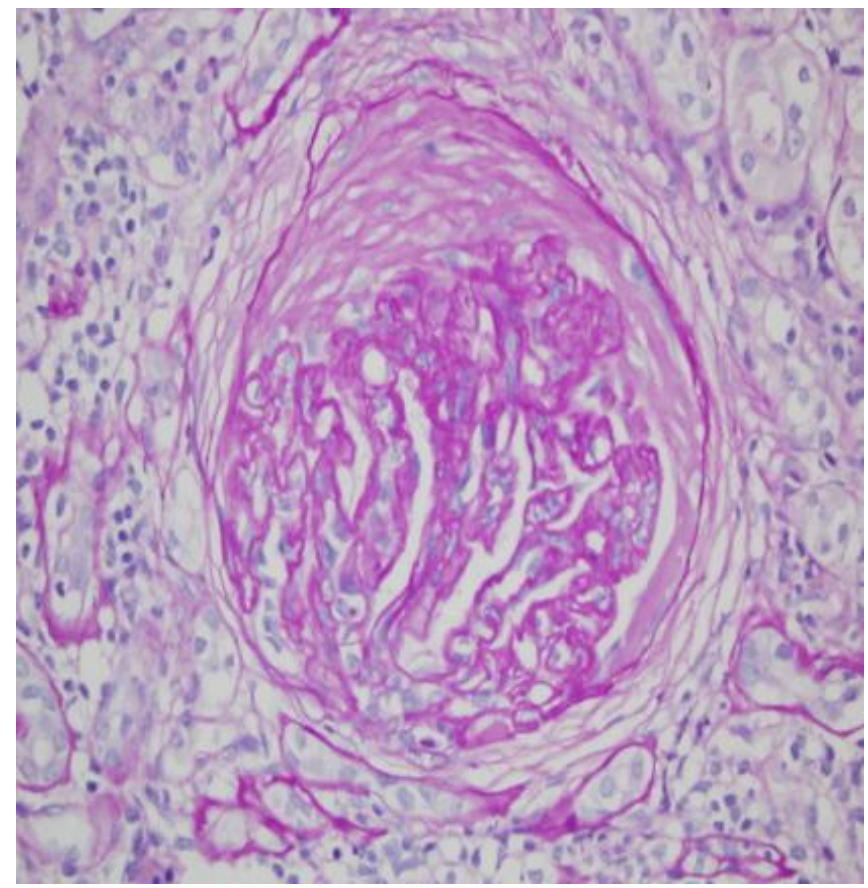

Kidney biopsy: crescentic glomerulonephritis

\section{Discussion}

The differential diagnosis of gastrointestinal involvement is quite difficult in patients with vasculitis. The concomitant infectious or inflammatory diseases of intestine such as Crohn's disease or the side effect of immunosuppressive drugs likewise corticosteroid therapy should be taken into consideration. Upper respiratory system involvement, rapidly progressive glomerulonephritis and ANCA positivity enabled us to suspect early. The upper respiratory tract is involved in more than $90 \%$ of cases of GPA and the nasal cavity and the paranasal sinuses are the most common sites of involvement in the head and neck area. Also the clinical or histological evidence of renal involvement are detected in about $80 \%$ of patients ${ }^{(4)}$. Although GIS involvement is rarely detected in GPA patients, gastrointestinal perforation is seen frequently in the small intestine with poor prognosis because of the high mortality rate $(46.7 \%)^{(5)}$. Anna Masiak et al. noticed that the histopathological changes in gastrointestinal tract revealed commonly nonspecific inflammation, not specific signs that confirm GPA ${ }^{(3)}$. Camilleri et al. suggested that this result may be attributed to superficially taken biopsy specimens that do not involve the small and medium diameter vessels located deeper in the intestine submucosa ${ }^{(6)}$. So non-specific histopathological findings should not removal us from the diagnosis.

Although the clear mechanism of ileal perforation is not known, an ischaemic and necrotizing process may be underlying cause in the pathogenesis. Up to $12 \%$ of deaths in GPA patients are related to gastrointestinal tract complications $^{(7)}$. Treatment modalities widely accepted for GPA are also effective in treating patients with gastrointestinal involvement. Patients with major complications may also require surgical intervention. Surgeons are rarely concerned with the management of GPA. Delayed diagnosis of GIS involvement or masked GIS symptoms by steroids may cause to fulminant sepsis, multi- organ failure and death.

\section{Conclusion}

Presenting with in a wide variety of clinical conditions leads to difficulties in establishing a timely diagnosis and appropriate treatment. Active inflammatory process in the gastrointestinal tract may occur as an initial manifestation of GPA and may cause to life threating complications. Clinicians should be aware for different presentations of disease to improve prognosis by early diagnosis and appropriate strong treatment.

\section{References}

1. Jennette JC. Nomenclature and classification of vasculitis: lessons learned from granulomatosis with polyangiitis Clin Exp Immunol. 2011;164:7-10.

2. Storesund B, Gran JT, Koldingsnes W. Severe intestinal involvement in Wegener's granulomatosis: report of two cases and review of the literature. $\mathrm{Br} \mathrm{J}$ Rheumatol. 1998;37:387-390.

3. Anna Masiak, Lukasz Zdrojewski, Zbigniew Zdrojewski, Barbara BulloPiontecka, Boleslaw Rutkowski. Gastrointestinal tract involvement in 


\section{JMSCR VoI||08||Issue ||07||Page 591-594||July}

granulomatosis with polyangiitis. Prz Gastroenterol. 2016;11:270-275.

4. A Greco, C Marinelli, M Fusconi, F Macri, A Gallo, A De Virgilio, G Zambetti, M de Vincentiis. Clinic manifestations in granulomatosis with polyangiitis.Int J Immunopathol Pharmacol 2016 Jun;29:151-159.

5. Takao Kiboshi, Kentaro Isoda, Koichi Furukawa, Tomoyuki Wakahara, Kenichiro Otani, Kayo Ueda. Granulomatosis with Polyangiitis Complicated with Gastrointestinal Perforation: A Case Report and Review of Literature Nihon Rinsho Meneki Gakkai Kaishi. 2017;40:382-386.

6. Camilleri M, Pusey C, Rees A. Gastrointestinal manifestations of systemic vasculitis. QJM. 1983;52:141-149.

7. Ushiyama O, Narikiyo T, Nagai Y et al. A case of Wegener's granulomatosis associated with refractory bowel granulomatous ulcers. Nihon Rinsho Meneki Gakkai Kaishi 1997;20:457-463. 